BULL. AUSTRAL. MATH. SOC.

\title{
FORCED OSCILLATIONS OF SOLUTIONS OF
}

\author{
PARABOLIC EQUATIONS
}

\author{
NORIO YOSHIDA
}

\begin{abstract}
Parabolic equations with forcing terms are studied and sufficient conditions are given that all solutions of boundary value problems are oscillatory in a cylindrical domain.

Recently there has been much interest in studying the oscillatory
\end{abstract} behaviour of solutions of parabolic equations with functional arguments. We refer the reader to Bykov and Kultaev [1], Kreith and Ladas [2] and the author [3]. However, forced oscillations have not been discussed.

In this paper we are concerned with the forced oscillation of solutions of the parabolic equation

$$
u_{t}-a(t) \Delta u+c(x, t, u(x, t), u(x, \sigma(t)))=f(x, t),(x, t) \in \Omega \times \mathbb{R}_{+},
$$

where $\Delta$ is the Laplacian in Euclidean $n$-space $\mathbb{R}^{n}, \mathbb{R}_{+}=[0, \infty)$ and $\Omega$ is a bounded domain in $\mathbb{R}^{n}$ with smooth boundary $a \Omega$. It is assumed that

$$
\begin{aligned}
& \left(\mathbb{A}_{1}\right) a(t) \text { is a nonnegative continuous function in } \mathbb{R}_{+} \text {and } f(x, t) \\
& \text { is a continuous function in } \bar{\Omega} \times \mathbb{R}_{+} ; \\
& \left(\mathrm{A}_{2}\right) c(x, t, \xi, n) \geqq 0 \text { for }(x, t) \in \Omega \times \mathbb{R}_{+}, \xi \geqq 0, \eta \geqq 0 \text {, and }
\end{aligned}
$$

Received 23 October 1986.

Copyright Clearance Centre, Inc. Serial-fee code: 0004-9729/87 $\$ A 2.00+0.00$. 


$$
\begin{aligned}
& c(x, t, \xi, n) \leqq 0 \text { for }(x, t) \in \Omega \times \mathbb{R}_{+}, \xi \leqq 0, n \leqq 0 ; \\
& \left(\mathbb{A}_{3}\right) \sigma(t) \text { is a continuous function in } \mathbb{R}_{+\cdot} \text { such that } \lim _{t \rightarrow \infty} \sigma(t)=\infty \text {. }
\end{aligned}
$$

Our objective is to present conditions which imply that every (classical) solution $u$ of ( 1 ) satisfying a certain boundary condition is oscillatory in $\Omega \times \mathbb{R}_{+}$in the sense that $u$ has a zero in $\Omega \times[t, \infty)$ for any $t>0$. We consider three kinds of boundary conditions:

$$
\begin{aligned}
& \left(\mathrm{B}_{1}\right) u=\phi \text { on } \partial \Omega \times \mathbb{R}_{+}, \\
& \left(\mathrm{B}_{2}\right) \frac{\partial u}{\partial v}=\psi \text { on } \partial \Omega \times \mathbb{R}_{+}, \\
& \left(\mathrm{B}_{3}\right) \frac{\partial u}{\partial v}+\mu u=0 \text { on } \partial \Omega \times \mathbb{R}_{+},
\end{aligned}
$$

where $\phi, \psi, \mu$ are continuous functions on $\partial \Omega \times \mathbb{R}_{+}, \nu$ denotes the unit exterior normal vector to $\partial \Omega$ and $\mu \geqq 0$ on $\partial \Omega \times \mathbb{R}_{+}$.

It is known that the first eigenvalue $\lambda_{1}$ of the eigenvalue problem

$$
\begin{gathered}
\Delta w+\lambda w=0 \text { in } \Omega \\
w=0 \text { on } \partial \Omega
\end{gathered}
$$

is positive and the corresponding eigenfunction $\Phi$ is positive in $\Omega$.

THEOREM 1. Assume that $\left(A_{1}\right)-\left(A_{3}\right)$ hold. Every solution $u$ of the problem (1), $\left(B_{1}\right)$ is oscillatory in $\Omega \times \mathbb{R}_{+}$if

$$
\begin{aligned}
& \lim _{s \rightarrow \infty} \inf _{\tilde{s}} \int_{\tilde{s}}^{s} \exp \left(\lambda_{1} A(t)\right)\left(-a(t) \int_{\partial \Omega} \phi \frac{\partial \Phi}{\partial \nu} d \omega+\int_{\Omega} f(x, t) \Phi d x\right) d t=-\infty \text {, } \\
& \limsup _{s \rightarrow \infty} \int_{\tilde{s}}^{s} \exp \left(\lambda_{1} A(t)\right)\left(-\alpha(t) \int_{\partial \Omega} \phi \frac{\partial \Phi}{\partial \nu} d \omega+\int_{\Omega} f(x, t) \Phi d x\right) d t=\infty
\end{aligned}
$$

for all Zarge $\tilde{s}$, where $A(t)=\int_{0}^{t} a(\tau) d \tau$.

Proof. Suppose to the contrary that there is a solution $u$ of the problem (1), (B) which has no zero in $\Omega \times\left[t_{0}, \infty\right)$ for some $t_{0}>0$. Let $u>0$ in $\Omega \times\left[t_{0}, \infty\right)$. Since $\lim _{t \rightarrow \infty} \sigma(t)=\infty$, there is a number $t_{1}$ such that $t_{1}>t_{0}$ and $\sigma(t) \geqq t_{0}\left(t \geqq t_{1}\right)$. Hence $u(x, \sigma(t))>0$ in 
$\Omega \times\left[t_{1}, \infty\right)$. From assumption $\left(A_{2}\right)$ we see that $c(x, t, u(x, t), u(x, \sigma(t))) \geq 0$ in $\Omega \times\left[t_{1}, \infty\right)$, and therefore

$$
u_{t}-a(t) \Delta u \leqq f(x, t) \text { in } \Omega \times\left[t_{1}, \infty\right) \text {. }
$$

Multiplying (2) by $\Phi$ and integrating over $\Omega$, we obtain

$$
\frac{d}{d t} \int_{\Omega} u \Phi d x-a(t) \int_{\Omega}(\Delta u) \Phi d x \leqq \int_{\Omega} f(x, t) \Phi d x, \quad t \geqq t_{1} .
$$

It follows from Green's formula that

(4)

$$
\begin{aligned}
\int_{\Omega}(\Delta u) \Phi d x & =\int_{\partial \Omega}\left(\frac{\partial u_{\Phi}}{\partial v}-u \frac{\partial \Phi}{\partial v}\right) d \omega+\int_{\Omega} u \Delta \Phi d x \\
& =-\int_{\partial \Omega} \phi \frac{\partial \Phi}{\partial v} d \omega-\lambda_{1} \int_{\Omega} u \Phi d x .
\end{aligned}
$$

Combining (3) with (4) yields

$$
\frac{d}{d t} \int_{\Omega} u \Phi d x+\lambda_{1} a(t) \int_{\Omega} u \Phi d x \leqq-a(t) \int_{\partial \Omega} \phi \frac{\partial \Phi}{\partial \nu} d \omega+\int_{\Omega} f(x, t) \Phi d x,
$$

which is equivalent to

(5) $\left(\exp \left(\lambda_{1} A(t)\right) U(t)\right)^{\prime} \leqq \exp \left(\lambda_{1} A(t)\right)\left(-a(t) \int_{\partial \Omega} \phi \frac{\partial \Phi}{\partial v} d \omega+\int_{\Omega} f(x, t) \Phi d x\right)$, where $A(t)=\int_{0}^{t} a(\tau) d \tau$ and $U(t)=\int_{\Omega} u \Phi d x$. Integrating (5) over $\left[t_{1}, s\right]$, we obtain

$$
\exp \left(\lambda_{1} A(s)\right) U(s)-\exp \left(\lambda_{1} A\left(t_{1}\right)\right) U\left(t_{1}\right)
$$

(6)

$$
\leqq \int_{t_{1}}^{s} \exp \left(\lambda_{1} A(t)\right)\left(-\alpha(t) \int_{\partial \Omega} \phi \frac{\partial \Phi}{\partial \nu} d \omega+\int_{\Omega} f(x, t) \Phi d x\right) d t .
$$

The hypothesis implies that the right hand side of $(6)$ is not bounded from below, and hence $\exp \left(\lambda_{1} A(s)\right) U(s)$ cannot be eventually positive. This contradicts the positivity of $\exp \left(\lambda_{1} A(s)\right) U(s)\left(s \in\left[t_{1}, \infty\right)\right)$. If $u<0$ in $\Omega \times\left[t_{0}, \infty\right), v \equiv-u$ satisfies 


$$
\frac{d}{d t} \int_{\Omega} v \Phi d x+\lambda_{1} a(t) \int_{\Omega} v \Phi d x \leqq-a(t) \int_{\partial \Omega}(-\phi) \frac{\partial \Phi}{\partial \nu} d \omega+\int_{\Omega}(-f(x, t)) \Phi d x
$$

Proceeding as in the case where $u>0$, we are led to a contradiction. The proof is complete.

A special case of the problem (1), $\left(\mathrm{B}_{1}\right)$ is the following:

$$
\begin{gathered}
u_{t}-\Delta u+c(x, t, u(x, t), u(x, \sigma(t)))=f(x, t),(x, t) \in \Omega \times \mathbb{R}_{+}, \\
u=0 \text { on } \partial \Omega \times \mathbb{R}_{+} .
\end{gathered}
$$

COROLLARY. Assume that $\left(A_{1}\right)-\left(A_{3}\right)$ hold. Every solution $u$ of the problem (7), (8) is oscilzatory in $\Omega \times \mathbb{R}_{+}$if

$$
\begin{aligned}
& \liminf _{s \rightarrow \infty} \int_{\tilde{s}}^{s} \exp \left(\lambda_{1} t\right)\left(\int_{\Omega} f(x, t) \Phi d x\right) d t=-\infty, \\
& \limsup _{s \rightarrow \infty} \int_{\tilde{s}}^{s} \exp \left(\lambda_{1} t\right)\left(\int_{\Omega} f(x, t) \Phi d x\right) d t=\infty
\end{aligned}
$$

for alz large $\tilde{s}$.

Proof. Since $A(t)=t$ and $\phi \equiv 0$, the conclusion follows from Theorem 1.

THEOREM 2. Assume that $\left(A_{1}\right)-\left(A_{3}\right)$ hold. Every solution $u$ of the problem (1), $\left(B_{2}\right)$ is oscillatory in $\Omega \times \mathbb{R}_{+}$if

$$
\begin{aligned}
& \lim _{s \rightarrow \infty} \inf \int_{\tilde{s}}^{s}\left(a(t) \int_{\partial \Omega} \psi d \omega+\int_{\Omega} f(x, t) d x\right) d t=-\infty, \\
& \lim \sup _{s \rightarrow \infty} \int_{\tilde{s}}^{s}\left(a(t) \int_{\partial \Omega} \psi d \omega+\int_{\Omega} f(x, t) d x\right) d t=\infty
\end{aligned}
$$

for azz large $\tilde{s}$.

Proof. Suppose that the problem $(1),\left(B_{2}\right)$ has a solution $u$ which has no zero in $\Omega \times\left[t_{0}, \infty\right)$ for some $t_{0}>0$. We may suppose that $u>0$ in $\Omega \times\left[t_{0}, \infty\right)$. As in the proof of Theorem 1 , we see that the inequality (2) holds. Integration of (2) over $\Omega$ gives

$$
\frac{d}{d t} \int_{\Omega} u d x \leqq a(t) \int_{\partial \Omega} \psi d \omega+\int_{\Omega} f(x, t) d x, \quad t \geqq t_{1} .
$$


Arguing as in the proof of Theorem 1, we are led to a contradiction. The proof is complete.

THEOREM 3. Assume that $\left(A_{1}\right)-\left(A_{3}\right)$ hold. Every solution $u$ of the problem (1), $\left(B_{3}\right)$ is oscillatory in $\Omega \times \mathbb{R}_{+}$if

$$
\begin{aligned}
& \liminf _{s \rightarrow \infty} \int_{\tilde{s}}^{s}\left(\int_{\Omega} f(x, t) d x\right) d t=-\infty, \\
& \lim \sup _{s \rightarrow \infty} \int_{\tilde{s}}^{s}\left(\int_{\Omega} f(x, t) d x\right) d t=\infty
\end{aligned}
$$

for all large $\tilde{s}$.

Proof. Let $u$ be a solution of $(1),\left(B_{3}\right)$, which has no zero in $\Omega \times\left[t_{0}, \infty\right)$ for some $t_{0}>0$. We may suppose that $u>0$ in $\Omega \times\left[t_{0}, \infty\right)$. Integrating (2) over $\Omega$ and taking into account $\left(B_{3}\right)$, we obtain

$$
\begin{aligned}
\frac{d}{d t} \int_{\Omega} u d x & \leqq a(t) \int_{\partial \Omega} \frac{\partial u}{\partial v} d \omega+\int_{\Omega} f(x, t) d x \\
& =-a(t) \int_{\partial \Omega} \mu u d \omega+\int_{\Omega} f(x, t) d x \leqq \int_{\Omega} f(x, t) d x, t \geqq t_{1} .
\end{aligned}
$$

The same argument as in the proof of Theorem 1 leads us to a contradiction.

EXAMPLE 1. We consider the problem

$$
\begin{gathered}
u_{t}-u_{x x}+e^{\pi / 2} u(x, t-\pi / 2)=2(\cos x) e^{t} \sin t,(x, t) \in(0, \pi / 2) \times \mathbb{R}_{+}, \\
-u_{x}(0, t)=0, u_{x}(\pi / 2, t)=-e^{t} \sin t, \quad t \in \mathbb{R}_{+} .
\end{gathered}
$$

Here $n=1, a(t) \equiv 1, \Omega=(0, \pi / 2), f(x, t)=2(\cos x) e^{t} \sin t$ and $\int_{\partial \Omega} \psi d \omega=-e^{t} \sin t$. We easily see that

$$
\begin{aligned}
& \int_{\tilde{s}}^{s}\left(\int_{\partial \Omega} \psi d \omega+\int_{\Omega} f(x, t) d x\right) d t \\
= & \int_{\tilde{s}}^{s} e^{t} \sin t d t \\
= & 2^{-1 / 2} e^{s} \sin (s-\pi / 4)+2^{-1} e^{\tilde{s}}(\cos \tilde{s}-\sin \tilde{s}) .
\end{aligned}
$$


Hence, we find that conditions (9) and (10) are satisfied. It follows from Theorem 2 that every solution $u$ of $(13),(14)$ is oscillatory in $(0, \pi / 2) \times \mathbb{R}_{+}$. One such solution is $u=(\cos x) e^{t} \sin t$.

EXAMPLE 2. We consider the problem

$$
\begin{gathered}
u_{t}-u_{x x}+e^{\pi / 2} u(x, t-\pi / 2)=(2 \cos x+1) e^{t} \cos t,(x, t) \in(0, \pi) \times \mathbb{R}_{+}, \\
-u_{x}(0, t)=u_{x}(\pi, t)=0, t \in \mathbb{R}_{+} .
\end{gathered}
$$

Here $n=1, a(t) \equiv 1, \Omega=(0, \pi)$ and $f(x, t)=(2 \cos x+1) e^{t} \cos t$. . Since

$$
\begin{aligned}
& \int_{\tilde{s}}^{s}\left(\int_{\Omega} f(x, t) d x\right) d t \\
= & \int_{\tilde{s}}^{s} \pi e^{t} \cos t d t \\
= & 2^{-1 / 2} \pi e^{s} \sin (s+\pi / 4)-(\pi / 2) e^{\tilde{s}}(\cos \tilde{s}+\sin \tilde{s}),
\end{aligned}
$$

conditions (11) and (12) are satisfied. Theorem 3 implies that every solution $u$ of (15), (16) is oscillatory in $(0, \pi) \times \mathbb{R}_{+}$. In fact, there is an oscillatory solution $u=(\cos x+1) e^{t} \cos t$ of the problem (15), (16).

\section{References}

[1] Ya. V. Bykov and T. Ch. Kultaev, "Oscillation of solutions of a class of parabolic equations", Izv. Akad. Nauk Kirgiz. SSR 6 (1983), 3-9 (Russian).

[2] K. Kreith and G. Ladas, "Allowable delays for positive diffusion processes", Hiroshima Math. J. 15 (1985), 437-443.

[3] N. Yoshida, "Oscillation of nonlinear parabolic equations with functional arguments", Hiioshima Math. J. 16 (1986), 305-314.

Department of Mathematics

Faculty of Engineering

Iwate University

Morioka, Japan. 